\title{
Affective state detection via facial expression analysis within a human-computer interaction context
}

\author{
Anas Samara ${ }^{1}\left(\mathbb{D} \cdot\right.$ Leo Galway $^{1} \cdot$ Raymond Bond $^{1} \cdot$ Hui Wang ${ }^{1}$
}

Received: 26 April 2017 / Accepted: 22 November 2017 / Published online: 4 December 2017

(c) The Author(s) 2017. This article is an open access publication

\begin{abstract}
The advancement in technology indicates that there is an opportunity to enhance human-computer interaction by way of affective state recognition. Affective state recognition is typically based on passive stimuli such as watching video clips, which does not reflect genuine interaction. This paper presents a study on affective state recognition using active stimuli, i.e. facial expressions of users when they attempt computerised tasks, particularly across typical usage of computer systems. A data collection experiment is presented for acquiring data from normal users whilst they interact with software, attempting to complete a set of predefined tasks. In addition, a hierarchical machine learning approach is presented for facial expression-based affective state recognition, which employs an Euclidean distance-based feature representation, conjointly with a customised encoding for users' self-reported affective states. Consequently, the aim is to find the potential relationship between the facial expressions, as defined by Paul Ekman, and the self-reported emotional states specified by users using Russells Circumplex model, in relation to the actual feelings and affective states. The main findings of this study suggest that facial expressions cannot precisely reveal the actual feelings of users whilst interacting with common computerised tasks. Moreover, during active interaction tasks more variation occurs within the facial expressions of participants than occurs within passive interaction.
\end{abstract}

Keywords Emotions $\cdot$ Human computer interaction $\cdot$ Facial expression

\section{Introduction}

In human-human interaction, one can intuitively predict the emotional state based on observations about persons facial expressions, body behaviour, and voice intonations (Karray et al. 2008). This ability is essential as humans often adapt their own behaviour based on such inferences. Correspondingly, in computer systems, various input modalities exist that can be utilised to acquire information about the user. Firstly, audio and visual-based input modalities, such as eye gaze tracking, facial expressions, body movement detection, and speech and auditory analysis may be employed as additional inputs. Secondly, physiological input modalities using sensor-based signals, such as electroencephalogram (EEG), galvanic skin response, and electrocardiogram can also be utilised. Alternatively, extra inputs may be gained

Anas Samara

samara-a@ulster.ac.uk

1 School of Computing, Ulster University, Belfast BT37 0QB, UK by interpreting user behaviour with the mouse movements, keyboard keystrokes and content viewing, or even a combination of all of these different modalities (Duric et al. 2002).

This paper describes a human-computer interaction (HCI) data collection study that has been developed to collect data from several input modalities, in order to be used for studying and modelling different characteristics of users across typical HCI context, where users interact with varied computer applications. Subsequently, the paper investigates automatic affective state techniques using analysis of facial expressions from static images, where an ensemble hierarchical classification approach with feature representation based on Euclidean distance has been used alongside various user-based self assessment mapping schemes. In addition, it aims to identify the affective states that users are experiencing whilst interacting with multiple graphical user interface-based applications and attempting different computerised tasks, in conjunction with validating related selfassessment and self-reporting techniques. Accordingly, the work presented herein contributes to the body of literature on automated facial expression recognition. 
The sections of the paper are organised as follows. Section 2 gives a brief background on the research area and related work. Section 3 introduces and discusses available facial expression datasets with relevant work on data collection. Section 4 outlines about the methodology employed by the techniques used in the associated experiments carried out, as well as the details of the data collection protocol utilised during the experiments. Section 5 presents the experiments together with their results. Section 6 concludes the paper with discussion on the results and a summary of the findings.

\section{Background and related work}

A variety of models have been developed for modelling innate human feelings; one such model is the Circumplex Model, proposed by Russell (1980), which asserts that the affective state of human feeling can be considered as a point in two dimensional space, as illustrated in Fig. 1. In particular, this model is composed of valence and arousal intensity dimensions, whereby valence represents the intrinsic attractiveness or averseness of an emotion, and can be presented as a pleasantunpleasant continuum (Frijda 1986), and arousal is the physiological and psychological state that activates the alertness, consciousness and attention as a reaction to stimuli, and can be presented as an activation-deactivation continuum (Coull 1998; Robbins 1997). Consequently, results from the experiments carried out and presented in this paper were analysed by means of valence and arousal separately and conjointly. Within the research literature, facial expression recognition is an active area of research despite the number of successes in this domain. For instance, studies where machine learning techniques trained on features extracted using different approaches achieved a reasonable classification accuracy such

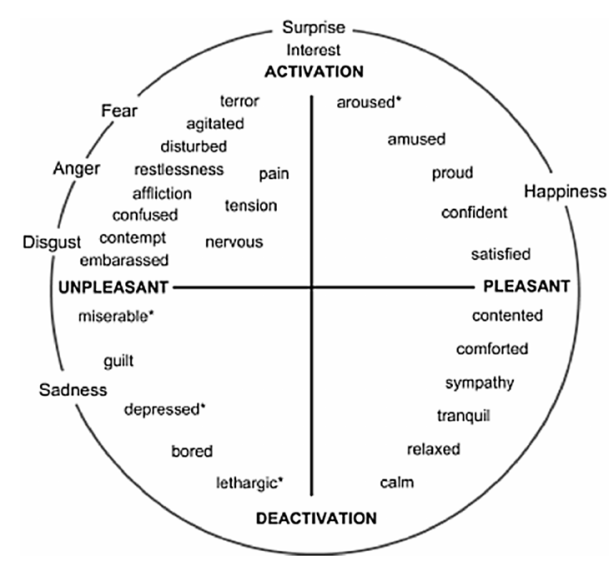

Fig. 1 Valence (pleasant-unpleasant continuum) versus arousal (activation-deactivation continuum), in the Circumplex Model (Russell and Lemay 2000) as the work of Liew and Yairi (2015) who achieved accuracy of $91.2 \%$ on CK-Plus dataset, using Support Vector Machine trained on Histogram of Oriented Gradients. Furthermore, recent progress with the evolution of Deep Learning techniques has produced a high classification accuracy of $96.76 \%$ on the same dataset, using Convolutional Neural Networks, as shown in the work presented by Lopes et al. (2017). Consequently, the work presented herein may be considered as comparable in terms of facial expression classification accuracy, whereas this work has been validated against multiple benchmark datasets.

\section{Datasets}

In terms of facial expression data, there are plenty of available datasets. For instance, Lucey et al. published a benchmark dataset entitled as Extended Cohn-Kanade (CK-Plus) database, which comprises 593 sequences taken from 123 subjects expressing the emotional states of: angry, contempt, disgust, fear, happy, sadness and surprise (Lucey et al. 2010). In a similar manner, Lundqvist et al. published the Karolinska Directed Emotional Faces dataset (KDEF), which consists of 4900 pictures captured from 70 subjects, whereby each subject acted seven different affective states: afraid, angry, disgust, happy, neutral, sad and surprise (Lundqvist et al. 1998). Furthermore, Siddiqi et al. had collected a dataset in which the expressions represent real life situations including emulated, semi-naturalistic, and naturalistic expressions (Siddiqi et al. 2017). Moreover, another multimodal dataset entitled DEAP, is a dataset for Emotion Analysis using EEG, Physiological and Video Signals for 32 subjects (frontal face video was recorded for only 22), who individually watched 401 -min music videos of different genres as a stimulus, to induce different affective and emotional states (Koelstra et al. 2012). Generally, such datasets could be used for the analysis of affective states, along with the assessment and self-reports made by the subjects themselves, such as using the Self-Assessment Manikin (SAM) scales (Bradley and Lang 1994), to typify the awareness of subjects' feelings. Consequently, the work presented herein investigates the relationship between facial expressions, as defined by Paul Ekman, and affective states. Particularly, the work focuses on self-reporting using Russell's Circumplex model that is used as a means to represent the actual experienced affective state (Russell 1980).

\section{Methodology}

\subsection{Feature extraction and distance-based representation}

Geometric-based techniques for facial expression analysis are based on locating the facial points and determining the 


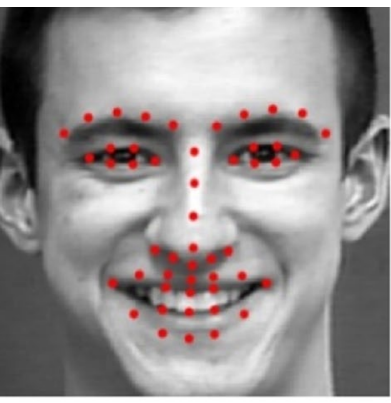

(a) Coordinate points

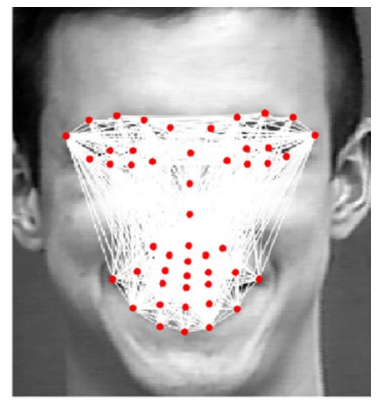

(b) Configural features
Fig. 2 Configural features of facial point coordinates

location and the shape of associated facial components, including the eyebrows, eyes, nose, lips and mouth. The study presented in this paper used the "Chehra" tool in order to extract the location of 49 facial points, as illustrated in Fig. 2a (Asthana et al. 2014). Chehra is a publicly available facial landmark detector based on discriminative facial deformable models, trained using a cascade of linear regressions. In addition, this tool is capable to locate the facial points automatically in real time and handle faces under uncontrolled natural setting. Subsequently, the detected landmarks are represented as the Cartesian coordinates of the extracted facial points, which produce a 98 -dimension feature vector that is used in the classification stage of the facial expression analysis pipeline, as mentioned in the experiment section.

Feature representation using Cartesian coordinates yielded a good classification accuracy. Nevertheless, this approach is not robust enough to permit recognition of facial expressions not provided in the training data. This is due to the fact that the constellation of these points varies among the myriad of facial shapes that comprise different facial morphologies (Salah et al. 2010). Therefore, we investigated another feature representation, which is primarily based on finding the configural features that represent intra-facial component distances, as shown in Fig. 2b (Martinez 2011). Subsequently, resultant features are represented by finding the Euclidean distances among all facial landmark points. Consequently, the facial expression is finally represented as a 1176-dimension feature vector, resulting from 49 Cartesian coordinate combinations (Samara et al. 2016).

\subsection{Hierarchical parallelised binary support vector machines}

Within the research literature, a range of classification techniques have been used in Affective Computing and emotion recognition using facial expressions as input vectors (Corneanu et al. 2016). In this study, we used a Support
Vector Machine (SVM), specifically the C-Support Vector with a linear kernel, available from the LIBSVM library developed at National Taiwan University (Chang and Lin 2001; EL-Manzalawy 2005). Furthermore, in this study we have investigated an approach that combines a set of SVM classifiers in order to improve the overall performance of the system. Initially, a number of labelled datasets (equal to the number of labels, i.e. classes) were produced from the original dataset. Accordingly, each dataset contains a pair of labels: either one of the labels from the original dataset, which corresponds to an emotional state, or others. Afterwards, each dataset generated is used to train a single binary classifier. Subsequently, a number of binary classifiers are trained individually on the individual two-class datasets, which comprise the first stage of the framework. The second stage of the framework then utilises a multi-class classifier that provides the final result from the overall classification task.

Thus, the resulting classification framework, Hierarchical Parallelised Binary Support Vector Machines (HPBSVM), operates in two stages as given in Fig. 3. During the first stage, binary SVM models are constructed from annotated data, with one model employed for each emotional state within the dataset. Furthermore, during the second stage, a multi-class SVM model is constructed to predict the state based on the combination of the decisions given from the binary SVM models of the first stage. The output from the first stage, which is a multiple components vector produced by the set of binary classifiers, represents the intermediate feature vector that is used to train a posteriori second stage multi-class classifier, entitled the Aggregation Classifier,

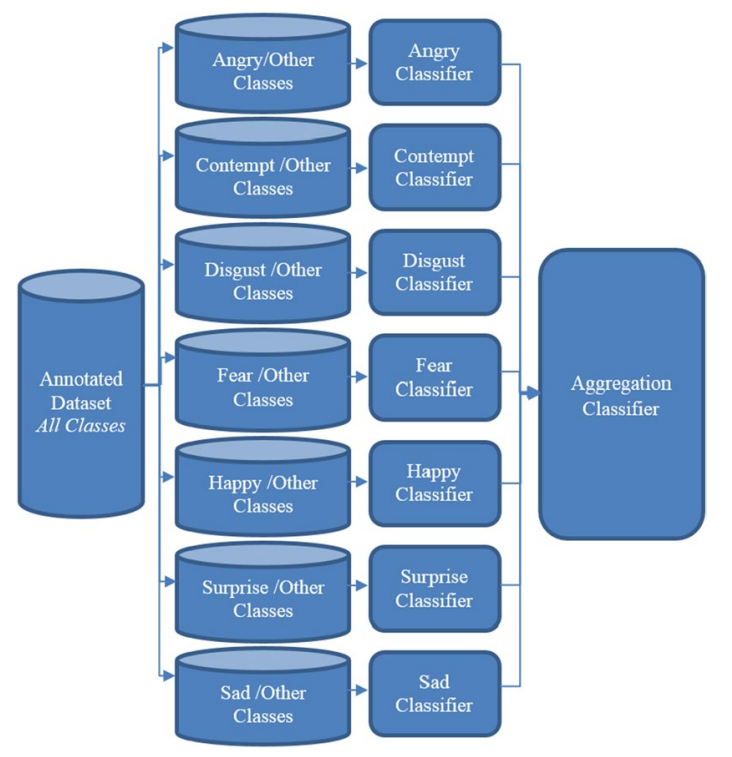

Fig. 3 Hierarchical parallelised binary support vector machines (HPBSVM) for emotion classification 
which gives the final decision on the detected emotional state. This approach facilitates more efficient and accurate classification of the whole system. The whole system benefits from the advantages of making some features more discriminative for specific classes. In other words, this framework decomposes the overall problem into smaller micro-decisions that are made by specialised classifiers, which are trained differently.

\subsection{Facial expression datasets}

Two different facial expression datasets have been selected for validating the suggested techniques: CK-Plus and KDEF, as they are considered to be benchmark datasets for facial expression analysis. These two datasets employ different labels for their annotated facial expressions. For CK-Plus, the labelling process was carried out over the peak frame using the Facial Action Coding System (FACS) (Ekman et al. 2002), and only 327 sequences meet the criteria to be labelled as a specific expression. For KDEF, the labels were given according to the emotion state that the subject was asked to attitudinise. In the experiments described later, our interest is to look at the facial expressions that may entail different affective states. Therefore, we have used another variation of CK-Plus by using neutral state snapshots. Therefore, three datasets have been utilised with the classifiers: CK-7, in which the sequences were divided into seven groups: angry (45), contempt (18), disgust (59), fear (25), happy(69), sadness (28) and surprise (83); CK-8, which expands upon CK-7 by incorporating 112 additional images annotated as neutral; $\mathrm{KDEF}$, in which we selected the frontal view of the snapshots, thereby producing an equal number of the seven states: afraid, angry, disgust, happy, neutral, sad and surprise.

\subsection{Data collection study}

The aim of the data collection study is to collect features from different input modalities, which is used to reason about users' affective states whilst interacting with common computer software and attempting to complete typical computer-based tasks. A total of 42 participants took part in the study. Participants were either staff or a student at Ulster University. There was no specific inclusion or exclusion criterion other than being a current student or staff member of the university, as the study is interested in identifying affective states in a generic HCI context, hence participants experiences could vary from novice to expert computer users.

\subsubsection{Material}

The material for the tasks used throughout the study can be classified into four main categories: (1) basic operating system tasks; (2) online shopping tasks; (3) Excel spreadsheet manipulation tasks, and (4) game-based tasks. Consequently, the themes of these had been chosen according to a study of computer usage statistics carried out by Beauvisage (2009), which presented the average distribution of individual weekly computer usage. Notably, the study showed that the four categories identified and selected for the study presented herein, occupy the highest percentages of time spent on computer usage for both households and individuals. Accordingly, the set of tasks represent active interaction with a computer in which the participant has a predefined task to carry out within a limited time of $5 \mathrm{~min}$ at most. Regarding the operating system task, the participant was asked within to change the desktop background, screen saver, time zone, and add a new input language to the system within the predefined time limit. For the online shopping task, the participant was asked to search online for a Tablet-PC with specific properties using their preferred Internet browser application. In terms of the spreadsheet manipulation task, the participant was asked to modify an existing Excel spreadsheet to insert new data into the existing records, sort the data in ascending order, use an aggregation function (i.e. average), and draw a line graph of the data. For the game-based task, the participant was asked to play a version of the arcade game Pacman (namely Deluxe Pacman 2- Release v16) for a total of $3 \mathrm{~min}$.

\subsubsection{Procedure}

Firstly, each participant was given an information sheet describing the flow of the experiment, and then he/she was asked to sign a consent form that his/her participation in the study is completely voluntary. At this stage, the participant commenced the first task within the designated time. On task completion, the participant was asked to complete SAM scales (Bradley and Lang 1994) to self-report the levels of valence and arousal during that task. During the experiment, the video of participant's face was recorded using a typical webcam placed at the top of the screen.

\subsection{Approach and analysis design}

This work aims to develop a validated technique for automatic classification of emotions from facial expressions by analysing video frames that were acquired whilst users attempted and interacted with each of the computer based tasks. Hence, the main objective of the classification is to model the affective states of users within a HCI context, and the association between the self-reporting and the facial expression. Subsequently, we categorised the tasks into two groups as active and passive interactions. Firstly, active interaction, which represents the situations where the user is working and undertaking an effort to carry out the task. 
These situations include: general operating systems tasks (tagged as $O S$ ), online shopping tasks (tagged as Online), and spreadsheet tasks (tagged as Excel), and entertainment task playing Pacman (tagged as Pacman). Secondly, passive interaction that represents situations where the user does not exert effort, which refers to the recordings contained within the DEAP dataset (Koelstra et al. 2012), where the subjects passively watched videos on Youtube without explicit interaction.

Moreover, as previously described, each participant carried out a self-reported assessment after performing each task using the SAM scale; this is the case in both the dataset obtained from the study and the DEAP dataset. Thus, each recorded video from each task is associated with a valence and arousal scores. Consequently, the relationship between facial expression percentages and the self-reported valence and arousal scores given by the participants for each task was further investigated. However, during analysis, rather than using the actual SAM ranges, i.e. (1.0-9.0), a mapping of the reported values into three ranges (low, medium and high) was used. Correspondingly, classification performance improves during supervised learning when the number of target classes is reduced (Aha 1992), especially when the combined classes have common properties and similarity, as is the case with our dataset. Therefore, a transformation was applied to the rounded values, as illustrated in Fig. 4, whereby values within the range (1.0-3.0) are labelled as low, values within the range (4.0-6.0) are labelled as medium, and values within the range (7.0-9.0) are labelled as high. In addition to this mapping, the labels corresponding to the self-reported values of valence and arousal have been used to represent the four quadrants of the Circumplex Model, as illustrated in Fig. 5, during the analysis carried out in this study.

\section{Results}

This section will summarise the experiment results achieved throughout the current study. Firstly, classification experiments applied on existing facial expression datasets. Secondly, percentages of facial expression that automatically applied on the video frames from the dataset obtained from the current work, which are recordings of subjects interacting with typical software interfaces. Thirdly, percentages of facial expressions across self-reporting ratings given by subjects, that are presumed to represent their actual feeling during each task, as well as, facial expression percentages according to combined ratings that are mapped to the Circumplex model quadrants. Experiments carried out herein used tenfold cross validation. In addition, a 95\% confidence interval of classification results has been used in order to

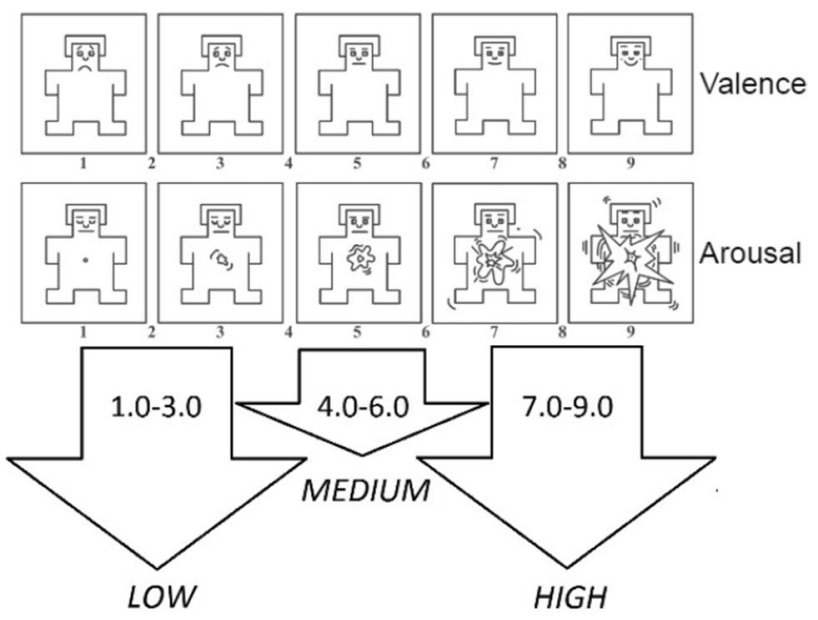

Fig. 4 Mapping from SAM scale value ranges into three labels [scores from (1.0-3.0) mapped to low, (4.0-6.0) mapped to medium, and (7.0-9.0) mapped to high]

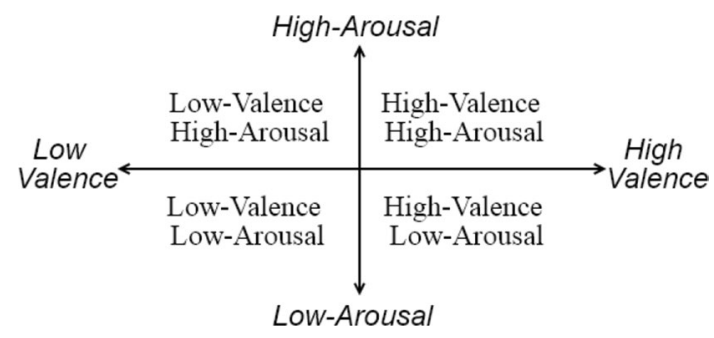

Fig. 5 Representation of Circumplex Model quadrants using combinations of Valence and Arousal score mappings, which utilises only the mappings corresponding to the High and Low labels

show the lower and upper limits along with the statistical significance of the results obtained.

\subsection{Expression datasets classification}

We have validated the performance of HPBSVM against the normal, single classifier scheme (i.e. SVM), using both the distance-based feature descriptor and a feature vector comprising Cartesian point coordinates. Basically, distancebased feature descriptor and HPBSVM outperforms point coordinates with SVM. Yet the improvement caused by the HPBSVM is substantial, with comparison to the improvement resulting from using distance-based descriptor. In brief, as given in Table 1, HPBSVM with distance-based descriptor achieves the higher accuracy rates across all three datasets. Consequently, this technique assures a credible result to be used with other data such as the one collected in our study and DEAP dataset. Therefore, we adopted this scheme in the experiments of next section. 


\subsection{Expression classification during human- computer interaction}

HPBSVM with distance-based feature was used to train two models using the validated datasets CK- 8 and KDEF, so that each resulting model can automatically classify the video frames of the recordings obtained during the computer-based tasks at a frequency of one frame per second. Figure 6 shows the percentages of facial expressions using the earlier mentioned trained models, which is achieved by automatically classifying the video frames of the user recording while carrying out the Online task. Figure 6a presents the expression percentages using a classification model that is trained on CK-8 dataset. Similarly, Fig. $6 \mathrm{~b}$ presents the expression percentages using a trained classifier using KDEF dataset.

As depicted in Fig. 6, different percentages of each expression have been found for both models. However, one can view these percentages differently by considering the fact that some expressions are much more precisely recognised than others. Generally, detecting states such as happy and surprise is comparably superior than detecting other states such as contempt, neutral, fear, angry, sadness and disgust, which is possibly due to the similarity in the geometric shape of these expressions (Samara et al. 2016). Moreover, the work presented in (Joho et al. 2009) underlined this type of grouping, by devising the pronounce level of the associated expressions, where these expressions belong to a low pronounced level. Therefore, the expression labels angry, contempt, disgust, fear and sadness from the CK-8 dataset used in our analysis can be combined together to represent the negative state. Likewise, the labels afraid, angry, disgust and sad from the KDEF dataset can be combined together to represent the negative state. Additionally, from the Circumplex Model it may be observed that there is a common aspect among these expressions, in that such negative labels occur on the negative side of the pleasantunpleasant continuum, e.g. the valence axis, as previously shown in Fig. 1. Therefore, the negative states grouping (to be within the negative state) applied to CK-8 results as well as KDEF results. After that, the resultant percentages obtained by averaging the output of the two trained models. Accordingly, the results depicted in Fig. 7, show percentages of each expression obtained from the videos recorded during each task using the average percentages across both the CK- 8 and KDEF trained models.

From Fig. 7, it can be observed that neutral and negative expressions occupy the highest percentages across the different tasks, with a greater number of negative expressions being shown during the tasks involving active interaction by participants. By contrast, in the passive interaction Youtube task, a greater level of neutral expression can be observed. Moreover, although a small percentage of happy expression may be observed in all tasks, within the Youtube task, the lowest percentage of happy expression is found. Therefore, it may potentially be surmised that, during active interaction tasks more variation occurs within the facial expressions of
Table 1 Classification accuracy of different datasets using pointcoordinates/distance-based with SVM/HPBSVM classification models

\begin{tabular}{lllll}
\hline Dataset & $\begin{array}{l}\text { Point-coordinates and } \\
\text { SVM (\%) }\end{array}$ & $\begin{array}{l}\text { Distance-based and } \\
\text { SVM }(\%)\end{array}$ & $\begin{array}{l}\text { Point-coordinates and } \\
\text { HPBSVM }(\%)\end{array}$ & $\begin{array}{l}\text { Distance-based } \\
\text { and HPBSVM } \\
(\%)\end{array}$ \\
\hline CK-7 & $80.75 \pm 4.28$ & $82.86 \pm 4.09$ & $96.02 \pm 2.24$ & $96.94 \pm 2.01$ \\
CK-8 & $76.76 \pm 3.95$ & $78.36 \pm 3.85$ & $95.22 \pm 2.06$ & $95.67 \pm 1.98$ \\
KDEF & $78.47 \pm 2.57$ & $81.84 \pm 2.42$ & $85.71 \pm 2.20$ & $96.22 \pm 1.22$ \\
\hline
\end{tabular}

Distance-based feature with HPBSVM outperforms point-coordinates with SVM with statistically significant improvement $(\mathrm{P}<0.001)$
Fig. 6 Percentages of expressions on CK-8 and KDEF trained models applied on recordings of Online task context respectively

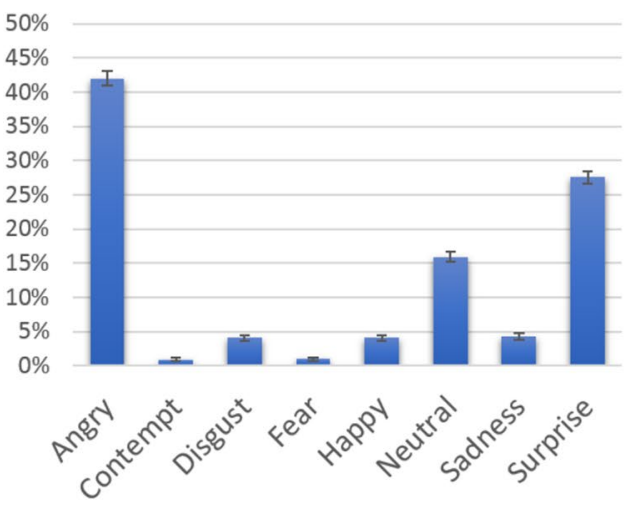

(a) CK-8 model output

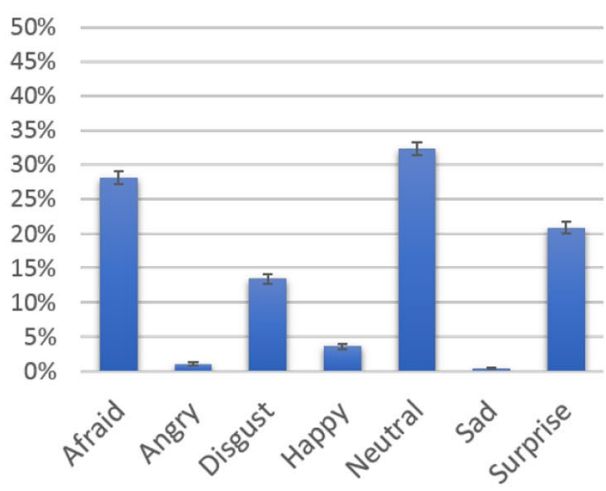

(b) KDEF model output 


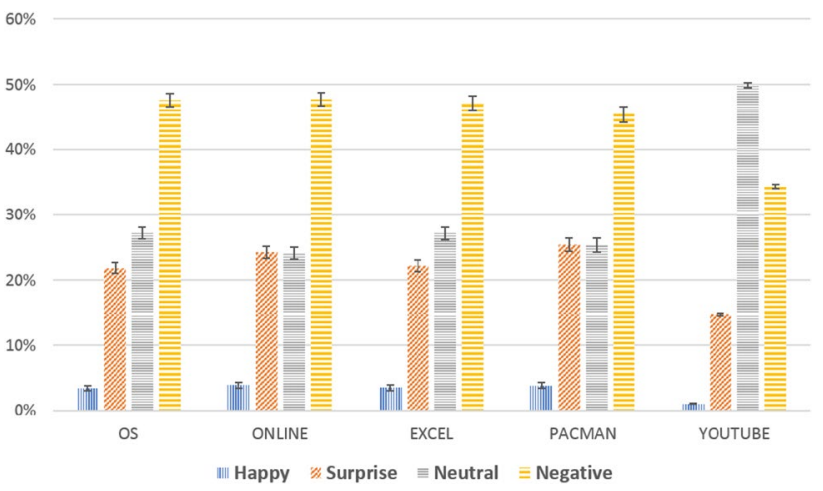

Fig. 7 Percentages of facial expressions across tasks by averaging outputs of two trained models on CK-8 and KDEF datasets

participants, than occurs within passive interaction tasks. However, such expressions might not reflect the actual feelings of the participants, as will be exposed later in this paper.

\subsection{Expressions versus reported valence and arousal}

We also compared the facial expression percentages with the ratings that were self-reported by subjects themselves for both valence and arousal. Table 2 gives the facial expression percentages obtained from averaging the results of the two trained models, across all the tasks (both active and passive), using the aforementioned mapping for the selfreported scores of valence and arousal respectively. In the same manner, combination of these scores as depicted in Fig. 5, has been aggregated versus facial expression percentages as given in Table 3. From the results given in the tables, it is apparent that the lowest percentage of frames, across all tasks, show the happy expression. However, somewhat surprisingly, tasks where participants self-reported high valence values correspond to the facial expression percentages where the happy expression is lowest too.

\section{Discussion and conclusion}

By merging low pronounced facial expressions such as contempt, neutral, fear, angry, sadness and disgust as a single facial expression label, negative, makes the analysis of the relationships between the facial expressions and affective states of users performing computer-based tasks much more persuasive. Especially, distinguishing between these facial expressions automatically is a non-robust job (Joho et al. 2009). We have explored a number of different analysis approaches in pursuit of understanding the potential relationships between self-reported affect and the corresponding recorded facial expressions over a number of tasks. Firstly, it was anticipated that there is a difference in the facial expression percentages between passive and active interaction contexts, due to the significant difference between the nature of the contexts.

Although a general inference is that both active and passive contexts are similar with regard to the relationships between the self-reported measures and the observed facial expressions, one difference found was that the facial expression alternation that occurs within an active context is marginally increased over that found within a passive context where an expression mostly remains as it is. In addition, there is a weak correlation, and inconsistency in some cases, between the individual and combined reported
Table 2 Facial expression percentages obtained based on classification of video frames using the average of the two trained models versus selfreported values of Valence $(\mathrm{Val})$ and Arousal (Aro)

\begin{tabular}{|c|c|c|c|c|c|c|c|c|c|}
\hline \multirow[t]{2}{*}{ Stimuli } & \multirow[t]{2}{*}{ Score } & \multicolumn{2}{|c|}{ Happy (\%) } & \multicolumn{2}{|c|}{ Surprise (\%) } & \multicolumn{2}{|c|}{ Neutral (\%) } & \multicolumn{2}{|c|}{ Negative (\%) } \\
\hline & & Val & Aro & Val & Aro & Val & Aro & Val & Aro \\
\hline \multirow[t]{3}{*}{ OS } & Low & 2.64 & 8.31 & 28.33 & 14.54 & 11.65 & 39.99 & 57.38 & 37.17 \\
\hline & Med & 3.72 & 2.53 & 20.07 & 15.65 & 33.84 & 32.56 & 42.38 & 49.26 \\
\hline & High & 3.38 & 3.76 & 18.12 & 31.91 & 17.27 & 17.23 & 61.23 & 47.1 \\
\hline \multirow[t]{3}{*}{ Online } & Low & 4.58 & 2.96 & 23.83 & 17.39 & 13.71 & 27.19 & 57.88 & 52.47 \\
\hline & Med & 4.08 & 4.51 & 21.91 & 23.7 & 25.83 & 25.93 & 48.18 & 45.85 \\
\hline & High & 2.53 & 3.08 & 32.94 & 26.85 & 27.62 & 20.42 & 36.91 & 49.65 \\
\hline \multirow[t]{3}{*}{ Excel } & Low & 0.81 & 3.02 & 27.69 & 25.75 & 26.49 & 34.44 & 45.01 & 36.79 \\
\hline & Med & 4.72 & 5.1 & 19.19 & 17.55 & 27.38 & 28.87 & 48.71 & 48.49 \\
\hline & High & 2.08 & 1.03 & 26.31 & 28.82 & 26.82 & 21.86 & 44.79 & 48.29 \\
\hline \multirow[t]{3}{*}{ Pacman } & Low & 2.75 & 4.67 & 18.44 & 32.45 & 23.82 & 31.25 & 54.99 & 31.63 \\
\hline & Med & 1.59 & 3.63 & 28.17 & 15.22 & 26.3 & 30.66 & 43.94 & 50.49 \\
\hline & High & 5.1 & 3.83 & 25.82 & 28.3 & 25.28 & 22.24 & 43.8 & 45.63 \\
\hline \multirow[t]{3}{*}{ Youtube } & Low & 0.5 & 0.97 & 16.84 & 15.05 & 47.62 & 54.83 & 35.03 & 29.15 \\
\hline & Med & 0.65 & 1.04 & 13.08 & 12.99 & 53.35 & 50.38 & 32.92 & 35.59 \\
\hline & High & 1.91 & 1.12 & 15.38 & 17.61 & 47.2 & 45.28 & 35.51 & 35.99 \\
\hline
\end{tabular}


Table 3 Facial expression percentages versus combination of subject self-reported values of Valence and Arousal together

\begin{tabular}{|c|c|c|c|c|c|}
\hline Stimuli & Valence/arousal & Happy (\%) & Surprise (\%) & Neutral (\%) & Negative (\%) \\
\hline \multirow[t]{4}{*}{ OS } & Low/low & 0 & 0 & 0 & 0 \\
\hline & Low/high & 0.13 & 41.59 & 3.25 & 55.03 \\
\hline & High/low & 0 & 0 & 0 & 0 \\
\hline & High/high & 2.43 & 20.56 & 27.25 & 49.76 \\
\hline \multirow[t]{4}{*}{ Online } & Low/Low & 0 & 0 & 0 & 0 \\
\hline & Low/high & 6.84 & 18.9 & 10.04 & 64.22 \\
\hline & High/low & 6.67 & 30 & 31.4 & 31.93 \\
\hline & High/high & 1.28 & 30.36 & 25.84 & 42.51 \\
\hline \multirow[t]{4}{*}{ Excel } & Low/Low & 1.95 & 15.61 & 51.46 & 30.98 \\
\hline & Low/High & 0.81 & 26.74 & 25.58 & 46.86 \\
\hline & High/Low & 6.65 & 18.97 & 42.36 & 32.02 \\
\hline & High/High & 1.59 & 32.34 & 21.55 & 44.52 \\
\hline \multirow[t]{4}{*}{ Pacman } & Low/Low & 2.45 & 46.57 & 12.25 & 38.73 \\
\hline & Low/High & 2.7 & 13.41 & 24.3 & 59.58 \\
\hline & High/Low & 6.49 & 32.15 & 39.23 & 22.12 \\
\hline & High/High & 4.66 & 30.15 & 22.39 & 42.79 \\
\hline \multirow[t]{4}{*}{ Youtube } & Low/Low & 0.61 & 16.5 & 56.9 & 26 \\
\hline & Low/High & 0.77 & 18.2 & 40.48 & 40.55 \\
\hline & High/Low & 1.47 & 13.68 & 52.02 & 32.83 \\
\hline & High/High & 1.48 & 18.65 & 46.21 & 33.67 \\
\hline
\end{tabular}

values of valence and arousal with facial expression found in the recordings. Subsequently, this potentially supports the hypothesis that facial expressions do not reflect the actual feelings of users within a HCI context. On the other hand, it may potentially indicate the fact that when participants attempted to assess and self-report their actual feelings, they were unable to accurately distinguish and report on different emotions and feelings. This point of view certainly agrees with the argument given in Rosalind (2003) that humans often do not know how to articulate their actual feelings and affective states due to ambiguity and mixed mental activities. In addition to the significant differences in valence and arousal values that were reported for the various tasks, simultaneously the same facial expressions were still portrayed.

Moreover, the results presented reveal that the accuracy of automatically detecting affective states using facial expression analysis, particularly within a HCI context, is not comparable to the accuracy achieved by facial expression analysis when acted and performed deliberately as commonly found in most work within the Affective Computing literature. Consequently, this could be due to the nature of the relationship between humans and computers. Ultimately, humans do not (currently) exchange emotions and feelings with machines in the same manner as they do when interacting with each other.

For these reasons, researchers look to other technologies for the purpose of detecting human emotions and states (Jaimes and Sebe 2007). Some of these technologies have very good recognition accuracy rates for certain states such as stress, which can be reflected through physiological responses such as heart rate and blood pulse volume, though they could be obtrusive and too noisy to be employed for generic HCI purposes. Additionally, some of the technologies may produce an extra effect on the users and cause additional feelings for humans, rather than detecting actual feelings they are attempting to measure (Hernandez et al. 2014). Therefore, HCI approaches and designs begin, especially within the last decade, to shift the focus from the behaviours and procedures of user experience, towards psychology and sociology concepts, which take into account human factors, emotions, cognitive aspects and individuals behaviours (Harper et al. 2008).

The work presented in this paper comprises deploying an approach for user modelling and affective state detection via facial expression analysis within HCI context. Employing a hierarchical ensemble model, the classification decision is decomposed into smaller micro-decisions that are individually made by specialised binary classifiers, thereby facilitating higher accuracy of the overall model. In addition, a data collection study, along with its corresponding findings from data analysis were discussed. The same analysis approach was also applied to data from another published dataset, namely DEAP dataset. Subsequently, from analysing video frames collected within different applications, it is concluded that facial expressions cannot precisely reveal the actual feelings of users. Experiments presented in this paper examined automatic affect recognition during 
common computer usage with results suggesting that facial expressions doubtfully indicate the actual feelings of users during interactions with computers. Consequently, work is needed to determine much more appropriate and effective techniques that reason upon users' experiences during interaction with computers in order to facilitate the generation of intelligent and adaptive systems. While the work presented within this paper investigates facial expression variation across different user interaction tasks, future work could undertake more in-depth experimentation and analysis of related cognitive load.

Although a lot of attention is paid towards deploying facial expression analysis in Affective Computing, the challenges encountered in these endeavours may not only be in terms of technical issues. On the contrary, one potential and significant challenge that may need to be addressed, for example, might be in humans' perception of computers; the perception that the computer is a machine that is a task oriented tool, which is inexpert to reason upon human feelings with the same intellect as that of another human. Thereupon, work is progressing in different directions within various disciplines to reinforce the link between the human and the computer.

Acknowledgements The authors gratefully acknowledge the Vice Chancellor's Research Scholarships of Ulster University for the generous financial support of the research project. As well as, we would like to thank The Health Informatics Society of Ireland for HISI Research Bursary Award given to support the current research. Additionally, we thank all the participants who accepted to take part in this study, their contribution is appreciated.

Open Access This article is distributed under the terms of the Creative Commons Attribution 4.0 International License (http://creativecommons.org/licenses/by/4.0/), which permits unrestricted use, distribution, and reproduction in any medium, provided you give appropriate credit to the original author(s) and the source, provide a link to the Creative Commons license, and indicate if changes were made.

\section{References}

Aha DW (1992) Generalizing from case studies: a case study. In: Proceedings of the 9th international conference on machine learning, pp $1-10$

Asthana A, Zafeiriou S, Cheng S, Pantic M (2014) Incremental face alignment in the wild. In: Proceedings of the IEEE conference on computer vision and pattern recognition, pp 1859-1866

Beauvisage T (2009) Computer usage in daily life. In: Proceedings of the SIGCHI conference on human factors in computing systems. ACM, pp 575-584

Bradley MM, Lang PJ (1994) Measuring emotion: the self-assessment manikin and the semantic differential. J Behav Ther Exp Psychiatry 25(1):49-59

Chang C-C, Lin C-J (2001) LIBSVM-a library for support vector machines. https://www.csie.ntu.edu.tw/ cjlin/libsvm/. Accessed Mar 2015

Corneanu CA, Simon MO, Cohn JF, Guerrero SE (2016) Survey on RGB, 3D, thermal, and multimodal approaches for facial expression recognition: history, trends, and affect-related applications. IEEE Trans Pattern Anal Mach Intell 38(8):1548-1568

Coull JT (1998) Neural correlates of attention and arousal: insights from electrophysiology, functional neuroimaging and psychopharmacology. Prog Neurobiol 55(4):343-361

Duric Z, Gray WD, Heishman R, Li F, Rosenfeld A, Schoelles MJ, Schunn C, Wechsler H (2002) Integrating perceptual and cognitive modeling for adaptive and intelligent human-computer interaction. Proc IEEE 90(7):1272-1289

Ekman P, Friesen WV, Hager JC (2002) Facial action coding system. Manual and investigator's guide. Research Nexus, Salt Lake City

El-Manzalawy Y, Honavar V (2005) WLSVM: integrating libsvm into WEKA environment. Software available at http://www.cs.iastate. edu/ yasser/wlsvm

Frijda NH (1986) The emotions, 1st edn. Cambridge University Press, Cambridge. ISBN-10: 0521316006, ISBN-13: 978-0521316002

Harper R, Rodden T, Rogers Y, Sellen A (2008) Being human: Humancomputer interaction in the year 2020. Microsoft Research, Cambridge

Hernandez J, Paredes P, Roseway A, Czerwinski M (2014) Under pressure: sensing stress of computer users. In: Proceedings of the SIGCHI conference on human factors in computing systems. ACM, pp 51-60

Jaimes A, Sebe N (2007) Multimodal human-computer interaction: a survey. Comput Vis Image Underst 108:116-134

Joho H, Jose JM, Valenti R, Sebe N (2009) Exploiting facial expressions for affective video summarisation. In: CIVR '09, p 1

Karray F, Alemzadeh M, Saleh JA, Arab MN (2008) Human-computer interaction: overview on state of the art. Int J Smart Sens Intell Syst 1(1):137-159

Koelstra S, Muhl C, Soleymani M, Lee J-S, Yazdani A, Ebrahimi T, Pun T, Nijholt A, Patras I (2012) Deap: a database for emotion analysis; using physiological signals. IEEE Trans Affect Comput 3(1):18-31

Liew CF, Yairi T (2015) Facial expression recognition and analysis: a comparison study of feature descriptors. IPSJ Trans Comput Vis Appl 7:104-120

Lopes AT, de Aguiar E, De Souza AF, Oliveira-Santos T (2017) Facial expression recognition with Convolutional Neural Networks: coping with few data and the training sample order. Pattern Recogn 61:610-628

Lucey P, Cohn JF, Kanade T, Saragih J, Ambadar Z, Matthews I (2010) The extended Cohn-Kanade dataset (CK+): a complete dataset for action unit and emotion-specified expression. In: IEEE computer society conference on computer vision and pattern recognition workshops (CVPRW). IEEE, pp 94-101

Lundqvist D, Flykt A, Öhman A (1998) The Karolinska directed emotional faces (KDEF). CD ROM from Department of Clinical Neuroscience, Psychology Section, Karolinska Institutet

Martinez AM (2011) Deciphering the face. In: IEEE computer society conference on computer vision and pattern recognition workshops

Picard RW (2003) Affective computing: challenges. Int J Hum Comput Stud 59(1):55-64

Robbins TW (1997) Arousal systems and attentional processes. Biol Psychol 45:57-71

Russell J, Lemay G (2000) Emotion concepts. In: Lewis MH-J (ed) Handbook of emotion. Guilford Press, New York

Russell JA (1980) A circumplex model of affect. J Personal Social Psychol 39(6): 1161-1178

Salah AA, Sebe N, Gevers T (2010) Communication and automatic interpretation of affect from facial expressions. Affect Comput Interact Psychol Cogn Neurosci Perspect 8:157-183

Samara A, Galway L, Bond R, Wang H (2016) Sensing affective states using facial expression analysis. In: Ubiquitous computing and ambient intelligence: 10th international conference, UCAmI 2016, San Bartolomé de Tirajana, Gran Canaria, Spain, November 
29-December 2, 2016, Proceedings, Part I 10. Springer, pp 341-352

Siddiqi MH, Ali M, Abdelrahman Eldib ME, Khan A, Banos O, Khan A, Lee S, Choo H (2017) Evaluating real-life performance of the state-of-the-art in facial expression recognition using a novel YouTube-based datasets. Multim Tools Appl. https://doi.org/10.1007/ s11042-016-4321-2 